\title{
Sunscreens in melanoma and skin cancer prevention
}

S kin cancers are the most common form of malignant disease in white populations

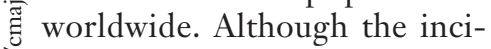
dence of cutaneous malignant melanoma is relatively low (Box 1 ), almost $15 \%$ of cases are fa$\stackrel{\circ}{\circ}$ tal. Squamous cell carcinoma

Box 1: Melanoma and skin cancer risk in the white population in Canada

Cancer

Estimated lifetime risk

Melanoma

$1.3 \%$ (about 1 in 76 )

Squamous cell carcinoma

$4.6 \%$ (about 1 in 20)

Basal cell carcinoma

$13.7 \%$ (about 1 in 7 )

Box 2: Summary of evidence from studies of the efficacy of sunscreen protection against malignant skin disease

Squamous cell skin cancer

Evidence

- Significantly fewer actinic keratoses among subjects randomly assigned to daily application of high-SPF sunscreens over a 1-year period (rate ratio $0.62,95 \%$ confidence interval $[\mathrm{Cl}] 0.54$ 0.71) (Thomson et al. N Engl J Med 1993;329:1147)

- Lower rate of new precancerous skin lesions among subjects randomly assigned to daily application of high-SPF sunscreens over a 2-year period (Naylor et al. Arch Dermatol 1995;131:170)

- Fewer new squamous cell carcinomas among subjects using sunscreen daily (incidence $1115 \mathrm{v}$. 1832 per 100 000, 95\% Cl 0.46-0.81); reduction evident after only 4 years of follow-up (Green et al. Lancet 1999;354:723)

Summary

Good evidence that sunscreens, when assiduously applied, can reduce risk of actinic keratoses and squamous cell skin cancer

Basal cell skin cancer

Evidence

- No reduced risk of initial basal cell carcinoma among subjects randomly assigned to daily sunscreen application and followed for $4-5$ years (Green et al. Lancet 1999;354:723)

Summary

No convincing evidence that sunscreen use will reduce risk of basal cell carcinoma

Melanoma

No adequate body of evidence free of confounding upon which to draw a conclusion as to the relation of sunscreens to melanoma, and hence no evidence of protective value and basal cell carcinoma, the 2 most common forms of nonmelanoma skin cancer, are usually treated surgically. They rarely cause death, but because they occur predominantly on sun-exposed sites such as the face, they can be disfiguring.

It is well established that sunlight can initiate and promote carcinogenesis and that excessive exposure is the main cause of both melanoma and nonmelanoma skin cancer. The proportion of melanoma in Canada caused by sunlight exposure is estimated to be more than $90 \%,{ }^{1}$ and this figure likely also applies to nonmelanoma skin cancers.

Because such a high proportion of malignant skin disease is attributable to sunlight exposure, skin cancer prevention messages and programs have become common in countries where the majority of the population has a lighter skin colour. In many of these programs, chemical sunscreens are suggested as a "last resort" in sun protection, to be used only if reductions in sunlight exposure cannot be achieved by the use of shade and protective clothing (long-sleeved shirts and long pants) and by limiting the time spent outdoors. Although the "cover-up" strategy may work well in countries like Australia, where sunlight abounds throughout the year, it is less effective in Canada, where sunshine is reliably available only 3 months each year. The question, then, for Canadian physicians making recommendations to their patients, is "What can sunscreens do and what can't they do?"

We know that all modern sunscreens with a high (15+) "sun protection factor" (SPF) give good protection against sunburn when properly applied. Sunburn is caused largely by ultraviolet B radiation (300-320 $\mathrm{nm}$ ), and older sunscreens also gave good protection from these wavelengths. There is some evidence that melanoma may also be re- lated to ultraviolet A exposure, but this is controversial since the studies showing such results use an animal model (fish of the $X i$ phophorus genus) with little resemblance to humans. ${ }^{2}$ Sunscreen manufacturers have responded with broad-spectrum sunscreens that provide substantial protection from ultraviolet $\mathrm{A}$ and $\mathrm{B}$ radiation.

The use of these new agents is not without concern, however. The results of a European trial of the use of unlabelled sunscreens of SPF 10 and 30 by people aged 18-24 demonstrated that those wearing the SPF 30 preparation spent more time in the sun per day (3.1 v. 2.6 hours) when on holiday than those using the SPF 10 compound. ${ }^{3}$ The amount of sunscreen used by each group was similar, as was the holiday duration. The degree of sunburn reported by each group was similar, so the higher SPF compound clearly provides better sunburn protection. However, if people spend more time in the sun to acquire the same degree of tan obtained when a lower SPF preparation is used, then this raises concerns about the long-term effects of increased exposure, largely because DNA damage can occur long before sunburn appears. Is there evidence that high-SPF, broadspectrum sunscreens provide protection against skin cancer? The answer appears to both "yes" and "no."

\section{Squamous cell carcinoma and basal cell carcinoma}

First, the good news. A number of trials have provided good evidence that, when applied consistently, sunscreens can realistically play a role in reducing the risk of squamous cell carcinoma (Box 2).

The story with basal cell carcinoma is less hopeful. Although studies are continuing, there is as yet no convincing ev- 
idence that sunscreen use reduces the risk of this type of carcinoma (Box 2).

\section{Melanoma}

Melanoma is a story unto itself. Because it is so much less common than either of the nonmelanoma skin cancers, it has not been feasible to conduct randomized trials aimed at sunscreen evaluation. Results from a randomized trial indicate that sunscreens attenuate the development of nevi in children over the short term (3 years), ${ }^{4}$ but it is not clear that this effect will persist, or that it predicts a reduced risk for melanoma later in life.

All other studies of the relation between sunscreen use and melanoma have been retrospective in nature (Box 3). Most showed no protective effects from sunscreen use, and some even indicated an increased risk for users. The results of these studies must be treated with caution. Most research conducted before the 1990s was designed to investigate the association between sun exposure, pigmentation characteristics and melanoma. As a consequence the data collected on sunscreen use was usually relatively cursory. To fairly evaluate the association, additional information is required, including whether a high-SPF sunscreen was used, whether it was applied in adequate amounts to attain the advertised SPF value, the frequency of sunscreen use, and the body sites it was applied to. Without accurate information on these variables, there is likely to be substantial misclassification in usage data with consequent dilution of the probability of finding a protective effect.

In addition to the information quality problems that affected earlier studies, serious potential problems with uncontrolled confounding are likely to have affected the results of even the more recent studies. Sunscreens are most commonly used by those with a strong propensity to burn in the sun because of fair skin. These same people are also at elevated risk of melanoma because of that same sun sensitivity, and techniques to measure sun sensitivity in population-based studies are unfortunately relatively crude. Thus it is highly likely that there is some degree of uncontrolled confounding from this factor in virtually all retrospective studies, and this may well be the reason why a number of the investigations show a direct rather than an inverse relation between use and melanoma risk (Box 3). Finally, there is evidence from previous etiologic studies that the degree of sun exposure in childhood and adolescence may be of particular importance in the later genesis of melanoma. If this is true, and effective sunscreens were not available to study subjects in this period, subsequent use will suggest a positive relation with risk.

Clearly these limitations present major problems in determining whether sunscreens have a role in melanoma prevention, and it is unlikely that they can be addressed through more retrospective investigations. A randomized trial beginning with the induction of healthy subjects would be the optimum method for moving forward, but such trials may not be practical for reasons of cost and study duration.

It is clear from this data that sunscreens cannot protect against all forms of skin cancer, and thus a rational strategy for skin cancer prevention must also include use of protective clothing. In addition, because all skin cancers are related to sun exposure, avoiding the sun is also likely to be beneficial. A practical approach is shown in Box 4.

It is important that these recommendations are accompanied by encouragement to participate in vigorous outdoor activity, with moderate sun exposure. With obesity on the rise, particularly among children, it would be counterproductive to encourage avoidance of outdoor activity. In addition, recent evidence suggests that vitamin D (largely generated through sun exposure) may be more important in health maintenance than we have traditionally believed.

\section{Richard P. Gallagher}

Cancer Control Research Program

B.C. Cancer Agency

Department of Health Care and

Epidemiology

Division of Dermatology

University of British Columbia

Vancouver, BC

\section{References}

1. Armstrong BK, Kricker A. How much melanoma is caused by sun exposure? Melanoma Res 1993;3:395-401.

2. Setlow RB, Grist E, Thompson K, Woodhead AD. Wavelengths effective in induction of malignant melanoma. Proc Natl Acad Sci USA 1993; 90:6666-70.

3. Autier P, Dore JF, Negrier S, Lienard D, Panizzon R, Lejeune FJ, et al. Sunscreen use and duration of sun exposure: a double-blind randomized trial. 7 Natl Cancer Inst 1999;91:1304-9.

4. Gallagher RP, Rivers JK, Lee TK, Bajdik CD, McLean DI, Coldman AJ. Broad-spectrum sunscreen use and the development of new nevi in white children. A randomized controlled trial. FAMA 2000;283:2955-60.

\section{Box 3: Reviews of sunscreen use and melanoma}

International Agency for Research on Cancer. IARC handbooks of cancer prevention. Vol. 5: Sunscreens. Lyon, France; IARC Press: 2001

Dennis LK, Beane Freeman LE, VanBeek MJ. Sunscreen use and risk for melanoma: a quantitative review. Ann Intern Med 2003;139:966-78

\section{Box 4: Summary recommendations}

1. Sunscreens should not be the first or sole agents used for skin cancer prevention, but should be used in conjunction with clothing to prevent overexposure.

2. Care must be taken not to use high SPF sunscreens to extend duration of exposure, such as prolonged sunbathing.

3. Daily spring and summer use of SPF $15+$ sunscreens on exposed skin should be encouraged among men and women working outdoors, as squamous cell carcinoma is most strongly related to chronic, cumulative sun exposure.

4. There are indications that childhood sun exposure may be particularly important in the generation of basal cell carcinoma and melanoma later in life. Because of this, the first 2 recommendations should be observed carefully by parents with young children.

Note: SPF = sun protection factor. 